\title{
Alcohol and hypertension: An analysis using The Health Survey for England 2014
}

\author{
Radu Grovu' ${ }^{1}$ and Jürgen Rehm, ${ }^{1,2,3,4}$ \\ ${ }^{1}$ Institute for Mental Health Policy Research, Centre for Addiction and Mental Health (CAMH), Toronto, Canada \\ ${ }^{2}$ Campbell Family Mental Health Research Institute, CAMH, Toronto, Canada \\ ${ }^{3}$ Addiction Policy, Dalla Lana School of Public Health, University of Toronto, Canada \\ ${ }^{4}$ Department of Psychiatry, Faculty of Medicine, University of Toronto, Canada \\ ${ }^{5}$ Epidemiological Research Unit, Technische Universität Dresden, Klinische Psychologie \& Psychotherapie, Dresden, Germany
}

\begin{abstract}
Aims: This study aims to model the risk relationships between alcohol consumption and hypertension, as alcohol is likely an important modifiable risk factor in treating hypertension and an important lifestyle variable to be taken into consideration by policy makers and physicians.
\end{abstract}

Design/Participants/Measures: This cross-sectional study uses data from the The Health Survey for England to perform a correlational analysis, as well as multinomial and binomial modeling to evaluate alcohol's impact on hypertension outcomes, all while controlling for relevant covariates (age, sex, smoking, exercise, body mass index, and education).

Findings: Findings indicate that alcohol consumption correlates with blood pressure and hypertension, yet the significance of these findings is weakened by large between-person variability and by confounding factors.

Conclusions: Based on these results, for the best cardiovascular health outcomes, we suggest that it is best to err on the side of caution and recommend, regarding alcohol intake, very limited (in the case of healthy patients) to no (for those with hypertension) alcohol consumption.

\section{Introduction}

Hypertension is a common medical condition in which blood pressure (BP) is elevated beyond safe levels. Patients with hypertension have a systolic BP $\geq 140 \mathrm{mmHg}$ and/or a diastolic BP $\geq 90 \mathrm{mmHg}$ (World Health Organization [WHO], 2013). This elevated BP puts those affected at risk of cardiovascular disease, which accounted for approximately 17.5 million deaths globally in 2012, nearly one third of total deaths (WHO, 2015). Hypertension alone in 2012 resulted in 9.4 million deaths worldwide from heart disease and stroke (WHO, 2015) and as a result is considered the primary individual risk factor for mortality worldwide, ranking third in terms of global burden of disease (WHO, 2014). In 2008, worldwide, approximately $40 \%$ of adults aged 25 and above had been diagnosed with hypertension; the number of people with the condition rose from 600 million in 1980 to 1 billion in 2008 (WHO, 2013). The increasing prevalence of hypertension is attributed to aging and behavioral risk factors, such as unhealthy diet, lack of physical activity, and a high body mass index (BMI), as well as harmful use of alcohol (WHO, 2015). This paper will focus on the relationship between alcohol and hypertension.

Alcohol is a major risk factor in terms of the global burden of disease (Wang et al., 2016). Alcohol consumption increases the risk for more than 200 disease and injury conditions (Rehm et al., 2010), including hypertension (WHO, 2013). In 2012, 5.9\% of all global deaths (approximately 3.3 million deaths) were attributable to alcohol, 33.4\% of which were due to cardiovascular disease and diabetes from alcohol consumption (WHO, 2014). By 2025 half of the WHO regions are expected to have increased alcohol consumption per capita (WHO, 2014). This indicates that alcohol will likely continue to have a significant global health impact and may be a greater burden on health outcomes in the future.

Alcohol has both acute and chronic effects on BP. Initially, drinking alcohol dilates blood vessels, lowering BP. Long-

Correspondence: Radu Grovu, Institute for Mental Health Policy Research, CAMH, 33 Russell Street, Toronto, Ontario, Canada, M5S 2S1. Telephone: 514 928 9013; Fax: 1416595 6033; Email: radu.c.grovu@gmail.com

Financial support: Institute for Mental Health Policy Research, Centre for Addiction and Mental Health, Canada

Declaration of interest: No conflicts of interest declared

Keywords: hypertension, blood pressure, alcohol, modeling, The Health Survey for England 2014 
term heavy alcohol use has the opposite effect, increasing $\mathrm{BP}$ as the body overcompensates for alcohol's depressant effects (Aguilera et al., 1999; Saunders, Beevers, \& Paton, 1981). Long-term heavy drinking has been shown to result in hypertension and other negative cardiovascular outcomedue to alcohol's toxic effects on organs and tissues, particularly the heart (Rehm, Gmel, Sempos, \& Trevisan, 2003).

Despite the damaging effects of alcohol, research indicates that drinking moderately protects against coronary heart disease (Fuchs, 2005), making it possible that moderate consumption may be beneficial for hypertensive people. A J-shaped dose-response relationship between BP and ingestion of alcohol has been described by multiple studies, meaning that drinking moderately may have a protective effect against negative cardiovascular outcomes and that the risk is highest for heavy drinkers (Plunk, SyedMohammed, Cavazos-Rehg, Bierut, \& Grucza, 2014; Reynolds et al., 2003; O’Keefe, Bybee, \& Lavie, 2007). This effect was found to be independent of several potential confounders such as BMI, education, smoking, and beverage type (Klatsky, 2004).

Because of the known association between alcohol and hypertension, this study aims to characterize their relationship and to quantify hypertension risk associated with increased alcohol consumption, as alcohol is likely an important modifiable risk factors in treating hypertension. These findings will serve to improve physicians' standards of care and will help to inform policy makers on alcohol's cardiovascular health impacts.

\section{Methods}

\section{Data Source: The Health Survey for England 2014}

The Health Survey for England (HSE) 2014 was collected from a population living in private households in England. It involved a stratified random sample of households in which adults and children were interviewed and visited by a nurse who gathered biometric data. The sample for the 2014 survey comprised 8,077 adults (ages 16 and over) and 2,003 children (ages 0 to 15). Since the data are based on a sample (not a census) of the population, the estimates are subject to sampling error.

\section{Inclusion and Exclusion Criteria}

In order to focus on the risk factors associated with primary hypertension, the full HSE dataset of 10,080 participant entries was not used. Participants were excluded from the dataset for the following reasons:

1. They were younger than 16 years old, the legal drinking age at which alcohol can be consumed in the company of an adult in the United Kingdom (UK Department of Government Services, n.d.).

2. They had no associated hypertension or alcohol consumption data.

For the above two reasons 5,488 participants were excluded (54\% of the original dataset).
3. They had no associated data concerning one or more of the confounders considered in this study: age, sex, smoking, education, exercise, BMI.

For this third reason, 952 participants were excluded (9\% of the original dataset). Data for these participants was determined to be missing completely at random using Little's test indicating that missing data will not likely affect the validity of our other statistical analyses.

The number of valid participant data entries after incomplete cases were removed was 3,640 (37\% of the original dataset), which is still a large sample and bodes well for the statistical power of our findings. To determine if there were significant differences between the original dataset and the subset used in this study, a chi-square comparison was performed. The $p$ values for hypertension and alcohol consumption between the two datasets were .51 and .33, respectively (both $>.05$ ). All other covariate $p$ values were also $>.05$, indicating no significant difference in the distribution of variables used between datasets.

\section{Dataset Analysis}

Our dataset consists of only categorical variables; therefore, to appropriately evaluate the relationship between alcohol and hypertension, two sets of models were built using the mutinom() and $\operatorname{glm}()$ functions from the nnet and stats packages in R. The first set included binomial models used to evaluate the effects of alcohol on a person's risk of having hypertension versus being normotensive (the reference group). The second set included multinomial models used to examine the effects of alcohol consumption on specific subcategories of hypertension, which includes the following:

- Hypertensive treated: Hypertensive people who take medication for their hypertension yet remain hypertensive.

- Hypertensive untreated: Hypertensive people who do not take medication for their hypertension.

For both model sets the reference group consisted of people who were normotensive. The outcome group for the binomial models was hypertension and for the multinomial models was one of the two specific subgroups of hypertension listed above. A model was selected from both sets after multiple considerations discussed in the "Selecting the Best Models" section, below. A mathematical description of the multinomial calculation used can be found in Appendix A. The significance threshold used in our analyses was $p<.05$.

\section{Confounders and Interactions}

Both the binomial and multinomial models built considered confounding variables likely to impact the association between alcohol consumption and the outcome variable, hypertension. The confounding variables considered include age, sex, smoking, exercise, BMI, and education, all of which are known risk factors for hypertension or have been associated with alcohol consumption (Fagard, 2006; Drøyvold, Midthjell, Nilsen, \& Holmen, 2005; Fukutomi \& Kario, 2010; Virdis, Giannarelli, Fritsch Neves, Taddei, \& 
Ghiadoni, 2010; Zimmerman \& Sullivan, 2013). Ethnicity, though associated with hypertension, was excluded from our analysis because the data sample was very skewed (more than any other variable), with $87 \%$ of participants identifying as white, and therefore would not allow for a fair assessment of ethnicity as a risk factor. Also, the confounders considered were limited in number to avoid overfitting of our statistical models.

The reference categories for all covariates considered can be found in Appendix B, and all of the response categories were unmodified and can be seen in Table 1. Exercise categories were derived from International Physical Activity Questionnaire tertiles of moderate or vigorous minutes of activity per week by the HSE. The only category modified from the original survey was alcohol consumption, which we regrouped to compare consumption with the National Institutes of Health (NIH) dietary guidelines (see Results section).

Our model sets also considered interactions between variables that could be justified by previous research. Justifications for the interactions considered can be found in Appendix C.

\section{Selecting the Best Models}

Four models were created for both the binomial and multinomial model sets.

1. Unadjusted Model: Incorporates only alcohol and hypertension variables.

2. Confounder Model: Incorporates alcohol, hypertension, and all confounders: age, sex, smoking, exercise, BMI, and education.

3. Confounder + Interactions Model: Incorporates all confounders and relevant interactions (Appendix C).
4. Significance Model: Incorporates only significant confounders and interactions found by evaluating the Confounder + Interactions Model using ANOVA.

For each set of models, prediction accuracy, AIC and BIC were used to select the most appropriate model for our analysis (Appendix D). The confounder model was found to be superior in both our binomial and multinomial model sets, since it has a low AIC and BIC while being the most accurate in predicting which hypertension class a person will fall into. Going from our unadjusted to our confounder model, the AIC and BIC decreased or remained unchanged while model accuracy improved, indicating that the confounder model fit our data well. Also, when interactions were added to the confounder model, none of them became significant after our ANOVA, indicating that it was a better fit to our data than the confounder + interactions model and the significance model.

\section{Defining Risk}

The Dietary Guidelines for Americans 2015-2020 defines moderate drinking as up to $12 \mathrm{~g}$ /day for women and up to $24 \mathrm{~g}$ /day for men (DeSalvo, Olson, \& Casavale, 2016). The NIH recommends no more than $48 \mathrm{~g}$ /day for men and $36 \mathrm{~g} /$ day for women in order to be considered low risk in terms of developing health conditions or alcohol abuse disorders (US Department of Health \& Human Services, 2010). In the United Kingdom, the Department of Health (n.d.) recommends no more than 21 units for men and 14 units for women per week or approximately $144 \mathrm{~g} /$ week for men and $96 \mathrm{~g} /$ week for women. All three guidelines agree on an ideal average of $24 \mathrm{~g} /$ day for men and approximately $12 \mathrm{~g} /$ day for women, which we used as our baseline or lowrisk group consumption. Exceeding this threshold puts participants in our at-risk group.

Table 1

Sample population distributions

\begin{tabular}{lcc}
\hline & \multicolumn{2}{c}{ Hypertension Status } \\
\cline { 2 - 3 } Covariates & Normotensive & Hypertensive \\
\hline Alcohol Consumption (g/day) & & $28(14.8 \%)$ \\
Former Drinker/Lifetime Abstainer & $496(14.4 \%)$ & $127(66 \%)$ \\
$\quad>0$ to $24 \mathrm{~g}$ & $2416(70 \%)$ & $16(8.3 \%)$ \\
$>24$ to $36 \mathrm{~g}$ & $264(7.7 \%)$ & $12(6.2 \%)$ \\
$>36$ to $<60 \mathrm{~g}$ & $184(5.3 \%)$ & $9(4.7 \%)$ \\
$\quad 60+\mathrm{g}$ & $88(2.6 \%)$ & $11(5.7 \%)$ \\
Age (years)* & & $103(54.0 \%)$ \\
16 to 34 & $730(21.0 \%)$ & $78(41.0 \%)$ \\
$\quad 35$ to 64 & $1839(53.0 \%)$ & $97(51.0 \%)$ \\
$\quad 65+$ & $879(25.0 \%)$ & $95(49.0 \%)$
\end{tabular}




\begin{tabular}{|c|c|c|}
\hline \multirow[b]{2}{*}{ Covariates } & \multicolumn{2}{|c|}{ Hypertension Status } \\
\hline & Normotensive & Hypertensive \\
\hline \multicolumn{3}{|l|}{ Exercise } \\
\hline Low & 1187 (34.0\%) & 77 (40.0\%) \\
\hline Medium & $1191(35.0 \%)$ & $63(33.0 \%)$ \\
\hline High & $1070(31.0 \%)$ & $52(27.0 \%)$ \\
\hline \multicolumn{3}{|l|}{ Education* } \\
\hline NVQ4/NVQ5 (Undergraduate Degree or Higher) & $976(28.0 \%)$ & $36(19.0 \%)$ \\
\hline Higher Ed (High School Degree) & $1003(29.0 \%)$ & $65(34.0 \%)$ \\
\hline NVQ1/NVQ2 (Below High School Degree) & $812(24.0 \%)$ & $41(21.0 \%)$ \\
\hline None & $657(19.0 \%)$ & $50(26.0 \%)$ \\
\hline \multicolumn{3}{|l|}{ Smoking Status } \\
\hline Never Smoked & $1829(53.0 \%)$ & $105(55.0 \%)$ \\
\hline Used to Smoke Occasionally & $202(5.9 \%)$ & $8(4.2 \%)$ \\
\hline Used to Smoke Regularly & $932(27.0 \%)$ & $56(29.0 \%)$ \\
\hline Current Smoker & $485(14.0 \%)$ & $23(12.0 \%)$ \\
\hline \multicolumn{3}{|l|}{ BMI* } \\
\hline Normal/Underweight & $1256(36.0 \%)$ & $39(20.0 \%)$ \\
\hline Overweight & 1297 (38.0 \%) & $73(38.0 \%)$ \\
\hline Obese & 895 (26.0 \%) & $80(42.0 \%)$ \\
\hline \multicolumn{3}{|l|}{ Treated for Hypertension* } \\
\hline No & $2880(84.0 \%)$ & $138(72.0 \%)$ \\
\hline Yes & $568(16.0 \%)$ & $54(28.0 \%)$ \\
\hline
\end{tabular}

Values are listed in the following format: $n$ (\% by column), with $n$ being the total number of participants in each category and the percentage expressed in terms of a value's proportion with respect to the total of its specific subcategory column (covariate columns add to $100 \%$ ). In total, there was a sample of 3,640 people, with 192 hypertensive participants and 3,448 normotensive participants. (* $p<.05$ using $t$ test with Bonferroni correction.)

\section{Results}

\section{Descriptive Analysis}

Of the covariates considered, alcohol consumption, age, education, and BMI significantly impacted the outcome of a participant being hypertensive or not. There is also a positive correlation between alcohol and multiple other variables, such as age, exercise, education, BMI, and hypertension. Proportionally, low-risk drinkers (0-24 g/day of alcohol) appear to have a lower prevalence of hypertension. This supports the known positive effect of moderate drinking on BP and hypertension (Breslow, Brown \& Chiapella, 2003).

\section{The Association Between Average Alcohol Consumption and Blood Pressure}

From Figure 1, we see that both systolic and diastolic BP are positively correlated with alcohol consumption. The increase in systolic and diastolic becomes significant $(p<.05)$ at $>24-36 \mathrm{~g}$ /day when comparing groups that drank to former drinkers and lifetime abstainers. We also notice that the relationship between alcohol consumption and BP does not have the common J-shaped curve known to model the relationship between alcohol consumption and the risk of cardiovascular disease morbidity and mortality (Breslow, Brown \& Chiapella, 2003). This indicates that cardioprotective effects of light to moderate alcohol consumption are likely not directly related to decreased BP. Other mechanisms, such as increased high-density lipoprotein cholesterol, increased fibrinolysis and decreased platelet aggregation, reduced inflammation, and increased insulin sensitivity likely play more significant roles (Breslow, Brown \& Chiapella, 2003). Although Figure 1 does show a positive correlation between alcohol consumption and BP, the average increase in BP is not very dramatic, and even those who consumed $>60 \mathrm{~g} /$ day had average BP values of $134 \mathrm{mmHg}$ systolic and $76 \mathrm{mmHg}$ diastolic, not high enough to be considered hypertensive. This indicates that not only alcohol but also other lifestyle factors need to be addressed in order to effectively lower dangerous BP levels.

\section{Alcohol versus Hypertension}

The association between alcohol consumption and our two hypertension subcategories is found to be significant ( $p<$ .05 ). As seen from Figure 2, alcohol is positively correlated with being hypertensive (both treated and untreated) and negatively correlated with being normotensive. Hypertension outcomes did not vary significantly $(p<.05)$ based on alcohol consumption when comparing men and women, so they are not shown separately. 
The association of alcohol with treatment-resistant hypertension indicates that alcohol may play a role in hypertension severity and treatment resistance. Patients whose BPs are controlled with four or more medications are considered to have treatment-resistant hypertension. People with treatment-resistant hypertension are at high risk for adverse cardiovascular events and are more likely than those with controlled hypertension to have a secondary incident, which is usually at least in part reversible by lifestyle changes such as reduced alcohol intake (Taler, 2005). The American Heart Association recommends cessation of heavy alcohol consumption when dealing with resistant hypertension to improve hypertension control (Chobanian, Bakris, \& Black, 2003). Heavy alcohol intake is associated with both an increased risk of hypertension and treatment-resistant hypertension. Wildman et al. (2005) showed that an intake of $>420 \mathrm{~g} /$ week increased the risk of either systolic or diastolic hypertension, and Henningsen et al. (1980) demonstrated that heavy drinkers with hypertension after a 2-year period were less likely to keep their BP under control than low-risk drinkers. In addition, Aguilera et al. (1999) studied a small group of heavy drinkers and found that reducing alcohol consumption reduced 24-hour ambulatory systolic and diastolic BPs by $7.2 \mathrm{mmHg}$ and $6.6 \mathrm{mmHg}$, respectively. Even though the overall trend for treatment-resistant hypertension is positive, the initial dip seen in the data is likely the result of high variability in these participants, along with a small sample size $(N=54$ with $n=17$ for those in the $60+\mathrm{g}$ /day group).

Figure 1

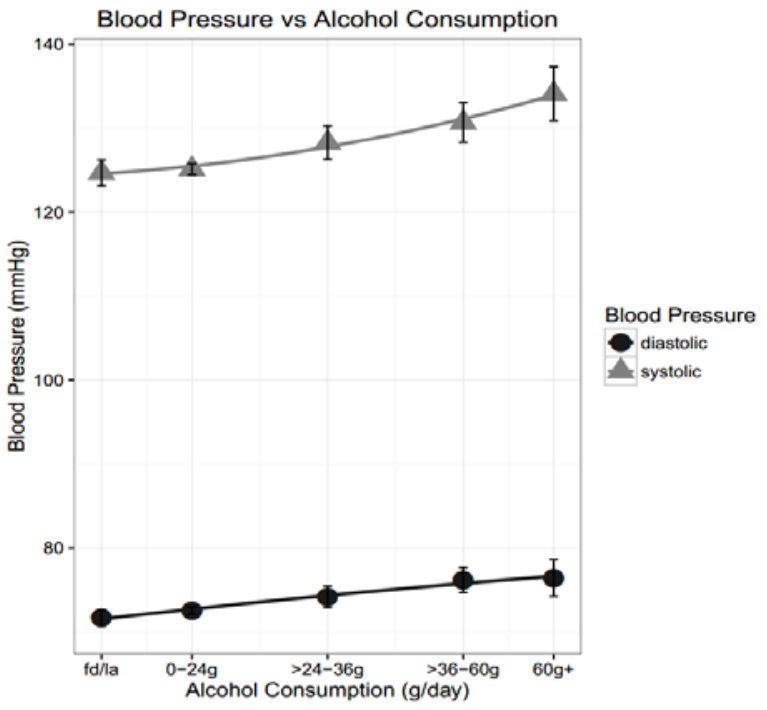

The $\mathrm{Y}$ axis represents a blood pressure average of 3 readings (see Appendix B). Error bars represent standard error with a $95 \%$ confidence interval. The alcohol consumption label of $\mathrm{fd} / \mathrm{la}$ indicates the level of consumption of a Former Drinker or Lifetime Abstainer.

Figure 2

The alcohol consumption label of fd/la indicates the level of consumption of a Former Drinker or Lifetime Abstainer. A linear regression for each hypertension outcome is plotted to show the general trends.
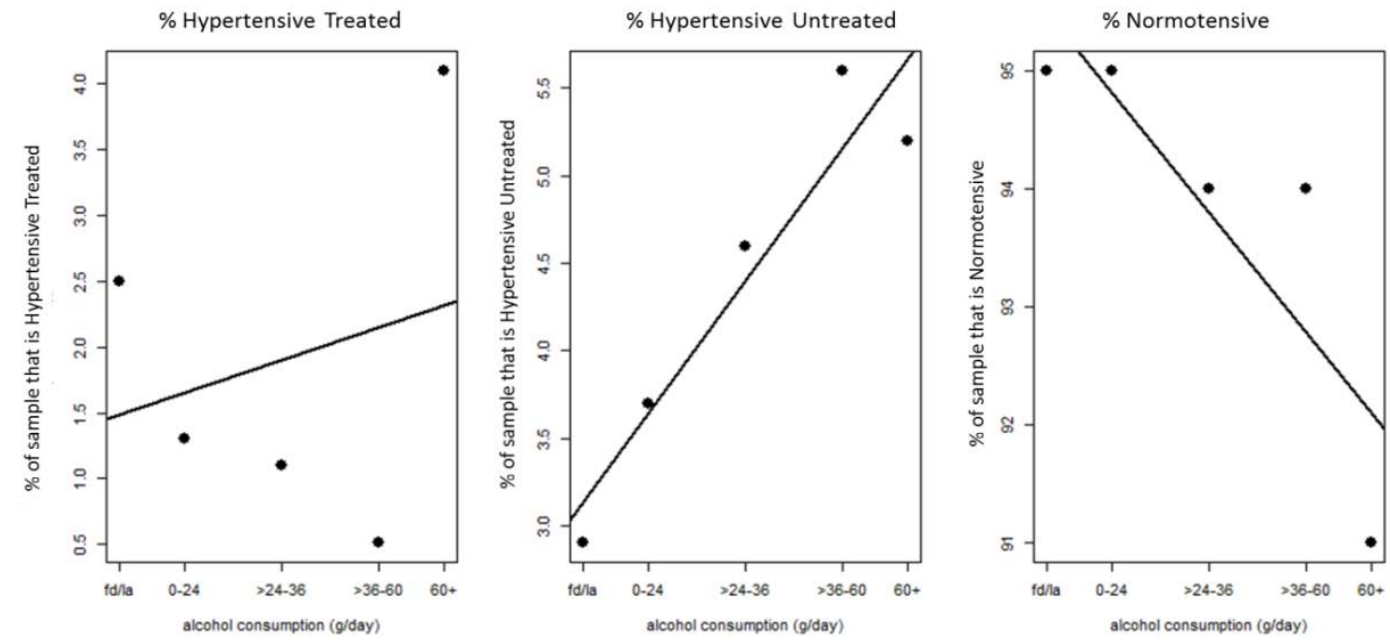

$R^{2}$ Hypertensive Treated $=0.57, R^{2}$ Hypertension Untreated $=0.90, R^{2}$ Normotensive $=0.72$ 
The positive correlation between alcohol consumption and untreated hypertension could be explained by alcohol's negative impact on compliance to treatment regimens in hypertensive patients (Jin, Sklar, Oh, \& Li, 2008). Noncompliance raises profound problems from a clinical perspective, since compliant patients generally have better health outcomes, and untreated hypertensives have a higher risk of hospitalization and death while also incurring, on average, higher medical expenditures (Nakamura et al., 2013).

Table 2 shows that there is no strong influence of riskassociated alcohol consumption on the incidence of hypertension. The difference in the prevalence of hypertension between low-risk and at-risk groups is also not statistically significant $(p>0.05)$.

\section{Relative Risk}

Table 3 shows a large amount of variance within each alcohol consumption category when evaluating hypertension risk. Our unadjusted binomial relative risk values show that low risk drinkers (0-24 g/day) had a significantly decreased risk of hypertension $(<1)$ compared to those in the high-risk group. This low-risk drinking benefit was also seen in our hypertensive treated group. Yet, when considering confounders in our adjusted relative risk, the benefit of this low-risk drinking is no longer present. The loss of significance when introducing covariates in both our binomial and multinomial models indicates that alcohol's protective effect is likely not primarily a result of drinking itself but may come from a related covariate.

Table 2

Percent of people with hypertension based on drinking risk category

\begin{tabular}{lcc}
\hline & \multicolumn{2}{c}{ Risk Groups } \\
\cline { 2 - 3 } Hypertensive & Low Risk & At Risk \\
\hline No & $2650(94.8 \%)$ & $798(94.5 \%)$ \\
Yes & $146(5.2 \%)$ & $46(5.5 \%)$ \\
Total & $2796(100 \%)$ & $844(100 \%)$ \\
\hline
\end{tabular}

Values are listed in the following format: $n$ (\% by column), with $n$ being the total number of participants in each category and the percentage being expressed in terms of a value's proportion with respect to the total of its specific column.

Table 3

Relative risk comparisons between models

Binomial Models

\begin{tabular}{lllll}
\hline Unadjusted & Alcohol Consumption (g/day) & Relative Risk & Lower CI & Upper CI \\
\hline & $>0$ to $24 \mathrm{~g}$ & 0.72 & 0.57 & 0.91 \\
& $>24$ to $36 \mathrm{~g}$ & 1.07 & 0.56 & 2.00 \\
& $>36$ to $<60 \mathrm{~g}$ & 1.16 & 0.56 & 2.27 \\
& $60 \mathrm{~g}+$ & 1.81 & 0.78 & 3.83 \\
\hline Adjusted & Alcohol Consumption (g/day) & Relative Risk & Lower CI & Upper CI \\
\hline & $>0$ to $24 \mathrm{~g}$ & 1.01 & 0.67 & 1.58 \\
& $>24$ to $36 \mathrm{~g}$ & 1.17 & 0.60 & 2.21 \\
& $>36$ to $<60 \mathrm{~g}$ & 1.13 & 0.54 & 2.24 \\
& $60 \mathrm{~g}+$ & 1.83 & 0.78 & 3.93 \\
\hline
\end{tabular}

\begin{tabular}{|c|c|c|c|c|}
\hline \multicolumn{5}{|c|}{ Multinomial Models } \\
\hline Unadjusted & Alcohol Consumption (g/day) & Relative Risk & Lower CI & Upper CI \\
\hline \multicolumn{5}{|c|}{ Hypertensive Treated } \\
\hline & $>0$ to $24 \mathrm{~g}$ & 0.52 & 0.27 & 0.90 \\
\hline & $>24$ to $36 \mathrm{~g}$ & 0.43 & 0.12 & 1.53 \\
\hline & $>36$ to $<60 \mathrm{~g}$ & 0.21 & 0.03 & 1.60 \\
\hline & $60 \mathrm{~g}+$ & 1.73 & 0.54 & 5.44 \\
\hline
\end{tabular}




\begin{tabular}{|c|c|c|c|c|}
\hline \multicolumn{5}{|c|}{ Multinomial Models } \\
\hline Unadjusted & Alcohol Consumption (g/day) & Relative Risk & Lower CI & Upper CI \\
\hline \multicolumn{5}{|c|}{ Hypertensive Untreated } \\
\hline & $>0$ to $24 \mathrm{~g}$ & 1.29 & 0.74 & 2.23 \\
\hline & $>24$ to $36 \mathrm{~g}$ & 1.63 & 0.76 & 3.47 \\
\hline & $>36$ to $<60 \mathrm{~g}$ & 2.00 & 0.89 & 4.38 \\
\hline & $60 \mathrm{~g}^{+}$ & 1.90 & 0.66 & 5.30 \\
\hline Adjusted & Alcohol Consumption (drinks/day) & Relative Risk & Lower CI & Upper CI \\
\hline \multicolumn{5}{|c|}{ Hypertensive Treated } \\
\hline & $>0$ to $24 \mathrm{~g}$ & 0.86 & 0.41 & 1.79 \\
\hline & $>24$ to $36 \mathrm{~g}$ & 0.57 & 0.14 & 2.29 \\
\hline & $>36$ to $<60 \mathrm{~g}$ & 0.26 & 0.03 & 2.17 \\
\hline & $60 \mathrm{~g}+$ & 4.49 & 1.08 & 18.6 \\
\hline \multicolumn{5}{|c|}{ Hypertensive Untreated } \\
\hline & $>0$ to $24 \mathrm{~g}$ & 1.24 & 0.70 & 2.17 \\
\hline & $>24$ to $36 \mathrm{~g}$ & 1.61 & 0.74 & 3.56 \\
\hline & $>36$ to $<60 \mathrm{~g}$ & 1.73 & 0.76 & 3.96 \\
\hline & $60 \mathrm{~g}+$ & 1.48 & 0.48 & 4.09 \\
\hline
\end{tabular}

The relative risk values here consider the outcome of being hypertensive (for binomial models) or being in a specific hypertension subcategory (multinomial models) relative to being normotensive. Unadjusted risk only considers alcohol consumption and the yes or no outcome of hypertension. Adjusted relative risk also considers age, sex, smoking, exercise, BMI, and education. The reference group for alcohol consumption consisted of individuals who were former drinkers or lifetime abstainers. CI represents a confidence interval of $95 \%$.

\section{Discussion}

In the United Kingdom, hypertension prevalence in 2014 was $32 \%$ among men and $27 \%$ among women (Craig, Fuller, \& Mindell, 2014). Also in the United Kingdom, $85 \%$ of the population had consumed alcohol at some point in their lives, according to the WHO Global Alcohol Report 2014 (WHO, 2014). Both alcohol and hypertension trends have stayed stable in the United Kingdom for the past few years, with neither varying significantly (WHO, 2014), indicating that the findings from our study will likely hold true for future drinkers.

The negative effects of alcohol, although well-known, are dependent on a wide variety of factors. As seen in Table 2, simply being in an at-risk drinking group did not significantly impact the outcome of hypertension. These unaccounted-for covariates likely also impacted the highly variable risk relationships between alcohol and hypertension (Table 3). The confounders considered in this study are likely not the only players in the alcoholhypertension relationship. Correlations between alcohol and health outcomes found in observational studies also require plausible biological mechanisms to be considered causal. Such mechanisms have been identified and include favorable effects of moderate alcohol consumption on some blood biomarkers, such as high-density lipoprotein cholesterol (Veenstra, et al., 1990). These positive mechanisms also have negative counterparts, such as the production of damaging reactive oxygen species and vascular desensitization. The impact of these mechanisms is further complicated by individual patient factors, such as genetic traits and lifestyle habits.
On top of the variability introduced by uncontrolled confounders, the data presented were also categorical in order to identify risk groups. This ignores the continuous nature of hypertension status and alcohol consumption, assuming homogeneity of risk within groups and decreasing the power of the study (Bennette \& Vickers, 2012). This may have also caused the large variability in relative risk seen in Table 3, especially since our exposure distribution to alcohol is asymmetric, with the vast majority of participants consuming 0-24 g/day. On the other hand, comparing results across studies is not difficult since many studies on alcohol's cardiovascular impact also place participants into similar drinking and hypertension categories (Husain, Ansari, \& Ferder, 2014; MacMahon, 1987; Puddey \& Beilin, 2006).

The HSE survey itself may have contributed to the lack of clear alcohol risk relationships. First, the survey data may suffer from systematic misclassification within the alcohol consumption categories. The common misclassification of occasional drinkers as abstainers may have negatively biased the health status of the reference group (former drinkers/lifetime abstainers) (Chikritzhs, Fillmore, \& Stockwell, 2009). Previous work has also shown that most self-reported drinking surveys underreport consumption, likely undermining the commonly reported health benefits of moderate drinking (Chikritzhs et al., 2009).

Our study may also suffer from the "sick quitter" effect, when former drinkers are mixed in with lifetime abstainers (Chikritzhs et al., 2009). Participants who gave up alcohol shortly before the survey were more likely to have significantly worse health profiles than those who are lifetime abstainers; this procedure affects the reference 
group and may change observed trends (Chikritzhs et al., 2009). Second, it is very difficult to completely rule out the influence of uncontrolled confounding variables on our results.

In summary, trends seen with BP and relative risk indicate that alcohol is associated with hypertension. The lack of significance when performing our statistical analysis between the two variables is due to a large variance within the sampled data and covariates that could not be controlled in our study.

\section{Conclusion}

Our results suggest that alcohol's effects on hypertension are complex and that contextual effects are important when evaluating their relationship. Even so, increased alcohol consumption has shown clear and significant $(p \leq .05)$ positive correlations with hypertension and should be a factor that clinicians discuss with their patients to help reduce its influence on future health risks the patient may face. Our cross-sectional study indicates that alcohol use should be considered in combination with other major lifestyle factors, such as exercise and well-suited treatment options, in order to best improve health outcomes for people suffering from hypertension. Future longitudinal evaluations of the link between alcohol and hypertension need to be done to clarify its relationship. The purported beneficial effects of low-dose alcohol consumption have been used as an argument against the implementation of strongly limiting population-level policies. Given the strong possibility that there are actually no cardioprotective effects and given the negative behavioral and non-hypertension related health effects of alcohol, policies to reduce alcohol-related harm should be considered-for example, erring on the side of caution via actions such as raising alcohol prices and restricting the physical availability of alcohol. Also, the current indication that moderate drinking is beneficial can confuse medical practitioners as to appropriate advice regarding alcohol consumption. From our results, we suggest that it is best to err on the side of caution and recommend very limited alcohol consumption (in the case of healthy patients) or no alcohol consumption (for those with hypertension) for the best cardiovascular health outcomes.

\section{Acknowledgments}

Special thanks to the UK Data Service for providing access to The Health Survey for England 2014 used in this study and to Afshin Vafaei, PhD, who provided insight into the statistical modeling processes used. Also thanks to Julian Wolf (University of California, Berkeley) who provided useful feedback when reviewing the manuscript.

\section{References}

Aguilera, M. T., de la Sierra, A., Coca, A., Estruch, R., Fernandez-Sola, J., \& Urbano-Marquez, A. (1999). Effect of alcohol abstinence on blood pressure assessment by 24-hour ambulatory blood pressure monitoring. Hypertension, 33(2), 653-657.

Bennette, C., \& Vickers, A. (2012). Against quantiles: Categorization of continuous variables in epidemiologic research, and its discontents. BMC Medical Research Methodology, 12(1), 1.

Bhupathy, P., Haines, C. D., \& Leinwand, L. A. (2010). Influence of sex hormones and phytoestrogens on heart disease in men and women. Women's Health, 6(1), 77-95.

Breslow, R., Brown, R., Chiapella P. (2003). State of the science report on the effects of moderate drinking. Bethesda, MD: National Institute on Alcohol Abuse and Alcoholism. Retrieved from https://pubs.niaaa. nih.gov/publications/moderatedrinking-03.htm

Cederbaum, A. I. (2012). Alcohol metabolism. Clinics in Liver Disease, 16(4), 667-685.

Chikritzhs, T., Fillmore, K., \& Stockwell, T. (2009). A healthy dose of scepticism: Four good reasons to think again about protective effects of alcohol on coronary heart disease. Drug and Alcohol Review, 28(4), 441444.

Chobanian, A., Bakris, G., \& Black, H. (2003). The seventh report of the Joint National Committee on Prevention, Detection, Evaluation, and Treatment of High Blood Pressure. JAMA, 289, 2560-2572.

Craig, R., Fuller, E., \& Mindell, J. (eds). Health Survey for England 2014: Health, Social Care and Lifestyles. Leeds, England: Health and Social Care Information Centre.

DeSalvo, K. B., Olson, R., \& Casavale, K. O. (2016). Dietary guidelines for Americans. JAMA, 315(5), 457-458.

Drøyvold, W. B., Midthjell, K., Nilsen, T. I. L., \& Holmen, J. (2005). Change in body mass index and its impact on blood pressure: A prospective population study. International Journal of Obesity, 29(6), 650-655.

El-Sayed, M. S., Ali, N., \& Ali, Z. E.-S. (2005). Interaction between alcohol and exercise. Sports Medicine, 35(3), 257-269.

Fagard, R. (2006). Exercise is good for your blood pressure: Effects of endurance training and resistance training. Clinical and Experimental Pharmacology and Physiology, 33(9), 853-856.

Flint, A. J., Rexrode, K. M., Hu, F. B., Glynn, R. J., Caspard, H., Manson, J. E., . . . Rimm, E. B. (2010). Body mass index, waist circumference, and risk of coronary heart disease: A prospective study among men and women. Obesity Research \& Clinical Practice, 4(3), e171-e181.

Fox, J., \& Hong, J. (2009). Effect displays in R for multinomial and proportional-odds logit models: Extensions to the effects package. Journal of Statistical Software, 32(1), 1-24.

Fuchs, F. D. (2005). Vascular effects of alcoholic beverages - Is it only alcohol that matters? Hypertension, 45(5), 851-852.

Fukutomi, M., \& Kario, K. (2011). Aging and hypertension. Expert Review of Cardiovascular Therapy, 8(11), 1531-9.

Gupta, S., \& Kapoor, S. (2010). Sex differences in blood pressure levels and its association with obesity 
indices: Who is at greater risk. Ethnicity \& Disease, 20(4), 370.

Henningsen, N., Ohlsson, O., Mattiasson, I., Trell, E., Kristensson, H., \& Hood, B. (1980). Hypertension, levels of serum gamma glutamyl transpeptidase and degree of blood pressure control in middle-aged males. Acta Medica Scandinavica, 207(1-6), 245-251.

Husain, K., Ansari, R. A., \& Ferder, L. (2014). Alcoholinduced hypertension: Mechanism and prevention. World Journal of Cardiology, 6(5), 245.

Jani, B., \& Rajkumar, C. (2006). Ageing and vascular ageing. Postgraduate Medical Journal, 82(968), 357362.

Jin, J., Sklar, G. E., Oh, V. M. S., \& Li, S. C. (2008). Factors affecting therapeutic compliance: A review from the patient's perspective. Therapeutics and Clinical Risk Management, 4(1), 269.

Jousilahti, P., Vartiainen, E., Tuomilehto, J., \& Puska, P. (1999). Sex, age, cardiovascular risk factors, and coronary heart disease - A prospective follow-up study of 14,786 middle-aged men and women in Finland. Circulation, 99(9), 1165-1172.

Klatsky, A. L. (2004). Alcohol and cardiovascular health. Integrative and Comparative Biology, 44(4), 324-328.

Kuerbis, A., Sacco, P., Blazer, D. G., \& Moore, A. A. (2014). Substance abuse among older adults. Clinics in Geriatric Medicine, 30(3), 629-654.

MacMahon, S. (1987). Alcohol consumption and hypertension. Hypertension, 9(2), 111-121.

Mainali, P., Pant, S., Rodriguez, A. P., Deshmukh, A., \& Mehta, J. L. (2015). Tobacco and cardiovascular health. Cardiovascular Toxicology, 15(2), 107-116.

Nakamura, K., Miura, K., Nakagawa, H., Okamura, T., Okuda, N., Nishimura, K., . . . Okayama, A. (2013). Treated and untreated hypertension, hospitalization, and medical expenditure: An epidemiological study in 314,622 beneficiaries of the medical insurance system in Japan. Journal of Hypertension, 31(5), 1032-1042.

O’Keefe, J. H., Bybee, K. A., \& Lavie, C. J. (2007). Alcohol and cardiovascular health: The razor-sharp double-edged sword. Journal of the American College of Cardiology, 50(11), 1009-1014.

Parnell, S. E., West, J. R., \& Chen, W.-J. A. (2006). Nicotine decreases blood alcohol concentrations in adult rats: A phenomenon potentially related to gastric function. Alcoholism: Clinical and Experimental Research, 30(8), 1408-1413.

Paton, A., \& McCune, A. (2015). Alcohol in the body. ABC of Alcohol, 12.

Plunk, A. D., Syed-Mohammed, H., Cavazos-Rehg, P., Bierut, L. J., \& Grucza, R. A. (2014). Alcohol consumption, heavy drinking, and mortality: Rethinking the j-shaped curve. Alcoholism: Clinical and Experimental Research, 38(2), 471-478.

Puddey, I. B., \& Beilin, L. J. (2006). Alcohol is bad for blood pressure. Clinical and Experimental Pharmacology and Physiology, 33(9), 847-852.

Rehm, J., Baliunas, D., Borges, G. L. G., Graham, K., Irving, H. M., Kehoe, T., . . . Taylor, B. (2010). The relation between different dimensions of alcohol consumption and burden of disease - An overview. Addiction, 105(5), 817-843.
Rehm, J., Gmel, G., Sempos, C. T., \& Trevisan, M. (2003). Alcohol-related morbidity and mortality. Alcohol Research \& Health, 140, C00-C97.

Reynolds, K., Nolen, J. D. L., Kinney, G., Sathya, B., He, J., \& Lewis, B. (2003). Alcohol consumption and risk of stroke: A meta-analysis. JAMA, 289(5), 579-588.

Saunders, J., Beevers, D., \& Paton, A. (1981). Alcoholinduced hypertension. The Lancet, 318(8248), 653656.

Taler, S. J. (2005). Treatment of resistant hypertension. Current Hypertension Reports, 7(5), 323-329.

Thirumalai, A., Rubinow, K. B., \& Page, S. T. (2015). An update on testosterone, HDL and cardiovascular risk in men. Clinical Lipidology, 10(3), 251-258. http://doi.org/10.2217/clp.15.10.

Thomasson, H. R. (2002). Gender differences in alcohol metabolism. In Recent Developments in Alcoholism (pp. 163-179). New York, NY: Springer.

UK Department of Government Services. (n.d.). Alcohol and Young People. Retrieved from https://www.gov.uk/alcohol-young-people-law

UK Department of Health. (n.d.). Updated Alcohol Consumption Guidelines Give New Advice on Limits for Men and Pregnant Women. Retrieved from https://www.gov.uk/government/news/new-alcoholguidelines

US Department of Health \& Human Services. (2004). The Health Consequences of Smoking: A Report of the Surgeon General. Atlanta, GA: US Department of Health and Human Services, Centers for Disease Control and Prevention, National Center for Chronic Disease Prevention and Health Promotion, Office on Smoking and Health.

US Department of Health and Human Services. (2010). Rethinking drinking: alcohol and your health (NIH Publication No. 10-3770). National Institute on Alcohol Abuse and Alcoholism, New York, NY.

Veenstra, J., Ockhuizen, T., van de Pol, H., Wedel, M., \& Schaafsma, G. (1990). Effects of a moderate dose of alcohol ön blood lipids and lipoproteins postprandially and in the fasting state. Alcohol and Alcoholism, 25(4), 371-377.

Virdis, A., Giannarelli, C., Fritsch Neves, M., Taddei, S., \& Ghiadoni, L. (2010). Cigarette smoking and hypertension. Current Pharmaceutical Design, 16(23), 2518-2525.

Wang, H., Naghavi, M., Allen, C., Barber, R. M., Bhutta, Z. A., Carter, A., ... Murray, C. J. (2016). Global, regional, and national life expectancy, all-cause mortality, and cause-specific mortality for 249 causes of death, 1980-2015: A systematic analysis for the Global Burden of Disease Study 2015. The Lancet, 388(10053), 1459-1544.

Wildman, R. P., Gu, D., Muntner, P., Huang, G., Chen, J., Duan, X., \& He, J. (2005). Alcohol intake and hypertension subtypes in chinese men. Journal of hypertension, 23(4), 737-743.

World Health Organization. (2013). A Global Brief on Hypertension: Silent Killer, Global Public Health Crisis. Geneva, Switzerland: World Health Organization. 
World Health Organization. (2014). Global Status Report on Alcohol and Health 2014. Geneva, Switzerland: World Health Organization.

World Health Organization. (2015). Global Status Report on Alcohol and Health 2015. Geneva, Switzerland: World Health Organization.
Zakhari, S. (2006). Overview: How is alcohol metabolized by the body?. Alcohol Research \& Health, 29(4), 245255.

Zimmerman, M. A., \& Sullivan, J. C. (2013). Hypertension: What's sex got to do with it? Physiology, 28(4), 234-244.

\section{Appendix}

\section{Appendix A: Multinomial Model Background (Fox \& Hong, 2009)}

The implementation of the multinomial logit model in the multinom function was taken from the nnet package in R. The multinomial logit model takes the following form:

$$
\begin{gathered}
u_{i j}=\frac{\exp \left(x_{i}^{\prime} \beta_{j}\right)}{1+\sum_{k=2}^{m} \exp \left(x_{k}^{\prime} \beta_{j}\right)} \\
u_{i 1}=\frac{1}{1+\sum_{k=2}^{m} \exp \left(x_{k}^{\prime} \beta_{j}\right)} \text { (for level 1) } \\
=1-\sum_{k=2}^{m} u_{i k}
\end{gathered}
$$

In this model $u_{i j}$ is the probability that the response variable is in the $\mathrm{j}^{\text {th }}$ of $\mathrm{m}$ total levels for observation $x_{i}^{\prime}$; is the $\mathrm{i}^{\text {th }}$ row of the model matrix X; and $\beta_{j}$ is a vector of $p$ regression coefficients pertaining to level $j$ of the response. The first level of the response is treated specially to ensure that $\sum_{j=1}^{m} u_{i j=1}$. This choice is arbitrary, in the sense that the model produces the same set of fitted probabilities regardless of the response level singled out for special treatment. The interpretation of the regression coefficients, however, depends upon the selection of baseline level for the response, because:

$$
\log \frac{u_{i j}}{u_{i 1}}=x_{i}^{\prime} \beta_{j} \text { for } \mathrm{j}=2 \ldots \mathrm{m}
$$

Thus, the coefficients represent effects on the log-odds of membership in level $\mathrm{j}$ versus level 1 of the response.

\section{Appendix B: Reference Categories}

When organizing the data, some values were taken directly from The Health Survey for England (HSE) 2014 and some we

\begin{tabular}{|c|c|}
\hline Survey Covariate & Subcategories \\
\hline Age & $\bullet \quad$ Age in years \\
\hline Sex & $\begin{array}{ll}\text { - } & \text { Male } \\
\text { - } & \text { Female }\end{array}$ \\
\hline Smoking & $\begin{array}{ll}\text { - } & \text { Never smoked cigarettes at all } \\
\text { - } & \text { Used to smoke cigarettes occasionally } \\
\text { - } & \text { Used to smoke cigarettes regularly } \\
\text { - } & \text { Current cigarette smoker }\end{array}$ \\
\hline Education & $\begin{array}{ll}\text { - } & \text { NVQ4/NVQ5 (Undergraduate Degree or Higher) } \\
\text { - } & \text { Higher Ed (High School Degree) } \\
\text { - } & \text { NVQ1/NVQ2 (Below High School Degree) } \\
\text { - } & \text { None }\end{array}$ \\
\hline Systolic/ Diastolic BP & $\begin{array}{l}\text { Omron Valid Mean Systolic BP: Each blood pressure measurement (both systolic } \\
\text { and diastolic) for study participants was taken three times using an Omron HEM } 907 \\
\text { in mmHG. }\end{array}$ \\
\hline
\end{tabular}
derived from the original survey values for our analysis. Details for both the direct and derived covariates are listed blow. Physiological variables including blood pressure (BP) and body mass index (BMI) were measured via nurse visit to the participant's household. For further details please consult the HSE 2014 documentation. 


\begin{tabular}{|c|c|}
\hline Survey Covariate & Subcategories \\
\hline Hypertension Treatment Groups & $\begin{array}{ll}\text { - } & \text { Normotensive untreated } \\
\text { - } & \text { Normotensive treated } \\
\text { - } & \text { Hypertensive treated } \\
& \end{array}$ \\
\hline Derived Covariates & Description \\
\hline Alcohol Consumption & $\begin{array}{l}\text { Alcohol from the survey was recoded in units/week from which we approximated } \\
\text { g/day using the following calculation: } \\
\text { g/day = units/week } * 1 \text { week/7 days } * 8 \mathrm{~g} / \text { unit } * 1 \text { drink } / 12 \mathrm{~g} \\
\text { These values were then arranged into the following subcategories in order to } \\
\text { compare with the National Institutes of Health's recommended consumption ranges: } \\
\qquad \quad>0 \text { to } 24 \mathrm{~g} \\
\text { - } \quad>24 \text { to } 36 \mathrm{~g} \\
\text { - } \quad>36 \text { to }<60 \mathrm{~g} \\
\text { - } 60+\mathrm{g}\end{array}$ \\
\hline BMI & $\begin{array}{l}\text { The original measurements were done using the participant's weight and height } \\
\text { (BMI = } \mathrm{kg} / \mathrm{m}^{2} \text { ) and included the following categories: } \\
\text { - } \quad \text { less than } 18.5 \\
\text { - } 18.5 \text { to less than } 25 \\
\text { - } \quad 25 \text { to less than } 30 \\
\text { - } \quad 30 \text { to less than } 35 \\
\text { - } \quad 35 \text { to less than } 40 \\
\text { - } \quad 40 \text { or more } \\
\text { These categories were combined due to a very small group sizes (incompatible with } \\
\text { building our regression models) to the following: } \\
\text { - } \quad \text { Normal/Underweight }(\leq 25) \\
\text { - } \quad \text { Overweight }(>25 \text { to } \leq 30) \\
\text { - } \quad \text { Obese }(30+)\end{array}$ \\
\hline Hypertension Status & $\begin{array}{l}\text { Hypertension status was derived from the treatment groups: } \\
\text { - } \quad \text { Normotensive } \\
\text { (normotensive treated + untreated) } \\
\text { - } \quad \begin{array}{l}\text { Hypertensive } \\
\text { (hypertensive treated }+ \text { untreated) }\end{array}\end{array}$ \\
\hline
\end{tabular}

For both sets of models (multinomial and binomial) the reference groups for the confounders are listed below:

\section{Covariate Reference Groups:}

- age: 16-24 years old

- sex: female

- alcohol: former drinker/ lifetime abstainer

- smoking: never smoked

- education: none

- exercise: low

- BMI: normal weight or underweight

- hypertensive: not hypertensive

\section{Appendix C: Valid Interactions}

\section{Interactions Considered:}

- alcohol: age, sex, smoking, exercise, body mass index (BMI)

- age: sex, smoking, exercise, BMI

- sex: exercise, BMI

- exercise: smoking, BMI 
- smoking: BMI

\section{Interaction: alcohol-age (yes)}

Sensitivity to alcohol's health effects increase with age. As people age, there is a decrease in the amount of water in the body, meaning that there is less water to dilute the alcohol that was consumed. This causes older adults to have a higher blood alcohol concentrations than younger people after consuming an equal amount of alcohol (Kuerbis, Sacco, Blazer, \& Moore, 2014). On top of this, metabolism of alcohol is slower in older people, allowing for stronger toxic effects (Kuerbis et al., 2014).

Older people are also more likely to be taking both prescription and non-prescription medications, some of which have negative side effects if combined with alcohol, such as aspirin, sleep medication, allergy medication, and pain killers (Kuerbis et al., 2014).

\section{Interaction: alcohol-sex (yes)}

Women appear to be more vulnerable than men to many adverse consequences of alcohol use. Women achieve higher concentrations of alcohol in the blood after drinking equivalent amounts (Thomasson, 2002). This is because women have less body water than men of similar body weight. In addition, women appear to eliminate alcohol from the blood faster than men. This finding may be explained by women's higher liver volume per kg of lean body mass, since alcohol is metabolized almost entirely in the liver (Thomasson, 2002).

\section{Interaction: alcohol-education (no)}

The toxic effects of alcohol have not yet been shown to vary with the person's educational status.

\section{Interaction: alcohol-exercise (yes)}

Most studies report a small increase in alcohol elimination rate in those who exercise regularly, perhaps due to increased body temperature or catecholamine release (Cederbaum, 2012). Exercise training also seems to reduce the extent of the oxidative damage caused by ethanol. Evidence generated from in vitro experiments and animal studies have suggested that ethanol administration decreases pyruvate-kinase and lactate dehydrogenase activities, which is countered by exercise (El-Sayed, Ali, \& Ali, 2005).

\section{Interaction: alcohol-smoking (yes)}

Smoking affects alcohol metabolism via its effects on gastric emptying, which is accelerated, and hence blood alcohol levels do not reach the same peak levels they would have in a non-smoker (Zakhari, 2006; Parnell, West, \& Chen, 2006).

\section{Interaction: alcohol-BMI (yes)}

Alcohol is not drawn into body fat as well as it is absorbed into lean muscle mass. Therefore, blood alcohol concentration is intensified in those with more body fat (Paton \& McCune, 2015). This results in alcohol having more damaging effects in those with higher BMI.

\section{Interaction: age-sex (yes)}

Estrogen improves endothelial cell function (Jousilahti, Vartiainen, Tuomilehto, \& Puska, 1999). The production of estrogen decreases after menopause, and this may change the female lipid metabolism toward a more atherogenic form, decreasing the high-density lipoprotein cholesterol level while increasing low-density lipoprotein and total cholesterol levels (Jousilahti et al., 1999). With age, sex hormone levels change, and this affects the impact of age on cardiovascular health. Among men and women, there are notable differences in body weight, height, body fat distribution, heart rate, stroke volume, and arterial compliance (Jani \& Rajkumar, 2006). In the very elderly, age-related large artery pulsatility and stiffness is more pronounced among women than men (Jani \& Rajkumar, 2006). This may be caused by women's smaller body size and arterial dimensions, which are independent of menopause (Jani \& Rajkumar, 2006).

\section{Interaction: age-education (no)}

The impact of age as a risk factor does not vary in its effects between those with varying educational levels directly without considering other exogenous factors.

\section{Interaction: age-exercise (yes)}

Exercise works to combat the negative effects of age on the cardiovascular system. Exercise increases cardiac output and combats vascular stiffness (El-Sayed et al., 2005; Cederbaum, 2012). A study by Cederbaum et al. showed that even after adjusting for age, VO2max remains greater among individuals participating in regular training compared to those who remain sedentary (Cederbaum, 2012). Other studies of older endurance-trained men demonstrate that frequent strenuous endurance exercise also preserves endothelial function (Cederbaum, 2012).

\section{Interaction: age-smoking (yes)}

Smoking causes endothelial dysfunction, fibrinolysis, lipid metabolism, and decreased oxygen supply to cardiovascular tissues, all of which have varying impact based on a person's age, because younger people have less accumulated damage to tissues than those who are older (US Department of Health \& Human Services, 2004). Smoking is a well-known age-compounding factor. It 
decreases endothelial nitric oxide synthase, causing vasoconstriction and increasing the activity of the sympathetic nervous system (nicotine increases the release of catecholamines), which in turn elevates heart rate and BP, resulting in wear and tear on the arterial walls (US Department of Health \& Human Services, 2004). Smoking also increases oxidative stress, which older bodies are less equipped to handle (Mainali, Pant, Rodriguez, Deshmukh, \& Mehta, 2015).

\section{Interaction: age-BMI (yes)}

A high BMI compounds the negative effects of age on the cardiovascular system. With age, the ratio of high-density lipoprotein and low-density lipoprotein fats changes in the body to favor low-density lipoprotein production, meaning that older people with a high BMI are more likely to suffer from plaque build-up in their vascular systems. Also, since age slows metabolism, BMI can more easily increase to unhealthy levels (Flint et al., 2010).

Interaction: sex-education (no)

It has yet to be shown that the effect of sex on cardiovascular health varies based on education.

Interaction: sex-exercise (yes)

Sex hormones, in particular estrogen, play a key function in maintaining myocardial mass (Bhupathy, Haines, \& Leinwand, 2010). Sex hormones also vary depending on a person's exercise regime (Bhupathy et al., 2010). Studies have also shown that low testosterone (which is increased by exercise) can also increase the risk of cardiovascular disease (Thirumalai, Rubinow, \& Page, 2010). Hence an interaction between the sex of a person and their level of exercise exists.

\section{Interaction: sex-smoking (no)}

Physiological effects of smoking have been shown to be independent of smokers’ sex (Mainali et al., 2015).

\section{Interaction: sex-BMI (yes)}

Body mass distributions and body fat levels vary between men and women. Men are more likely to accumulate fat in the abdominal area as opposed to the lower body and hence are at higher risk of cardiovascular damage due to plaque build-up around the heart and major vessels (Gupta \& Kapoor, 2010).

Interaction: education-exercise (no)

There is no evidence that varying levels of education affects the positive impact of exercise on cardiovascular health.

Interaction: education-smoking (no)

There is no evidence that varying levels of education affects the negative impact of smoking on cardiovascular health.

Interaction: education-BMI (no)

There is no evidence that varying levels of education affects the negative impact of BMI on cardiovascular health.

Interaction: exercise-smoking (yes)

Exercise and smoking have counteracting effects on cardiovascular health; thus their effects vary depending on the other's status.

Interaction: exercise-BMI (yes)

Exercise and BMI have counteracting effects on cardiovascular health; thus their effects vary depending on the other's status.

Interaction: smoking-BMI (yes)

Smoking and a high BMI have synergistic effects, both negatively impacting cardiovascular health.

\section{Appendix D: Model Comparisons}

\begin{tabular}{ccccc}
\hline Binomial Models: & Unadjusted Model & Confounder Model & $\begin{array}{c}\text { Confounder }+ \\
\text { Interactions Model }\end{array}$ & Significance Model \\
\hline Prediction Accuracy & 0.93 & 0.95 & 0.95 & 0.94 \\
AIC & 1510 & 1456 & 1548 & 1469 \\
BIC & 1514 & 1537 & 2131 & 1630 \\
& & & Confounder + & Significance Model \\
\hline & & & 0.93 \\
Multinomial Models: & Unadjusted Model & Confounder Model & Interactions Model & 1476 \\
Prediction Accuracy & 0.92 & 0.95 & 0.95 & 1630 \\
\hline
\end{tabular}

